\title{
Critical amplitude ratios of the Baxter-Wu model
}

\author{
Lev N. Shchur ${ }^{a, *}$, Wolfhard Janke ${ }^{b}$ \\ ${ }^{a}$ Landau Institute for Theoretical Physics, 142432 Chernogolovka, Russia \\ ${ }^{\mathrm{b}}$ Institut für Theoretische Physik and Centre for Theoretical Sciences (NTZ), Universität Leipzig, \\ Postfach 100 920, 04009 Leipzig, Germany
}

Received 7 July 2010; accepted 23 July 2010

Available online 3 August 2010

\begin{abstract}
A Monte Carlo simulation study of the critical and off-critical behavior of the Baxter-Wu model, which belongs to the universality class of the 4-state Potts model, was performed. We estimate the critical temperature window using known analytical results for the specific heat and magnetization. This helps us to extract reliable values of universal combinations of critical amplitudes with reasonable accuracy. Comparisons with approximate analytical predictions and other numerical results are discussed.
\end{abstract}

(c) 2010 Elsevier B.V. All rights reserved.

\section{Introduction}

One of the central results of the theory of phase transitions and critical phenomena is the formulation of the universality hypothesis [1,2]. According to the theory, all systems with the same dimensionality, the same symmetry of the ordered phase and the same number of order parameters are described by the same set of critical exponents at the critical point. Additionally, thermodynamic functions vary with temperature in such a way that some combinations of their amplitudes take the same values for all systems within a universality class [3]. For many systems, critical exponents are known by approximate methods (field theoretical perturbation theory, series expansions, Monte Carlo simulations) and have been derived exactly in some cases, mainly for two-dimensional models [4-9]. The extensive research in the last 50 years gives strong sup-

\footnotetext{
* Corresponding author.

E-mail addresses: lev@landau.ac.ru (L.N. Shchur), Wolfhard.Janke@itp.uni-leipzig.de (W. Janke).
} 
port for the universality hypothesis in the context of the critical exponents. ${ }^{1}$ At the same time, the issue of critical amplitude ratios was checked only for a few models [14].

Special interest in the properties of universality classes derives from cases in which the singular behavior is complicated by logarithmic corrections [15]. There are some systems which belong to the same universality class but whose off-critical behavior may be modified by logarithmic corrections. This is the case for the universality class of the two-dimensional 4-state Potts model. The model, which gives the name to this universality class, the 4-state Potts model [16] contains logarithmic corrections to the critical behavior [17-20] of thermodynamic quantities. For example, the free energy $F$ and the magnetization $M$ in the ordered phase behave at criticality as $[17,18]$

$$
\begin{aligned}
& F(\tau) \approx A_{4 \mathrm{p}}|\tau|^{2-\alpha}[\ln (-\tau)]^{\alpha_{l}}, \\
& M(-\tau) \approx B_{4 \mathrm{p}}(-\tau)^{\beta}[\ln (-\tau)]^{\beta_{l}},
\end{aligned}
$$

where $\alpha=2 / 3, \beta=1 / 12, \alpha_{l}=-1, \beta_{l}=-1 / 8$, and $\tau=1-T_{c} / T$ is a measure of the distance of the temperature $T$ to the critical temperature $T_{c}$.

Another two-dimensional model, which belongs to the universality class of the 4-state Potts model, is the Baxter-Wu model [21]. For this model it is known exactly [21-24] that close to the critical temperature, the free energy and the magnetization in the ordered phase behave as

$$
\begin{aligned}
& F(\tau) \approx A_{\mathrm{bw}}|\tau|^{2-\alpha}, \\
& M(-\tau) \approx B_{\mathrm{bw}}(-\tau)^{\beta},
\end{aligned}
$$

with the same values for the critical exponents as in (1) and (2), but without logarithmic corrections to the singular behavior.

A possible explanation for the difference in the off-critical behavior of the Baxter-Wu and 4-state Potts models is that for some unknown reason the coefficient behind the logarithmic correction is zero for the Baxter-Wu model [17,18], although the leading critical behavior for both models seems to be described by the same fixed point in the renormalization-group space [25].

Critical amplitudes are not universal and one should not expect that the free-energy amplitudes $A_{\mathrm{bw}}$ of the Baxter-Wu model and $A_{4 \mathrm{p}}$ of the 4-state Potts model are equal. At the same time, some combinations of the amplitudes are universal. For example, the ratio of the free-energy amplitude $A_{\mathrm{bw}}(+)$ in the high-temperature phase of the Baxter-Wu model to the amplitude $A_{\mathrm{bw}}(-)$ in the low-temperature phase equals unity, as well as the ratio of the corresponding amplitudes of the 4-state Potts model, $A_{4 \mathrm{p}}(+) / A_{4 \mathrm{p}}(-)=1$. This is a consequence of the duality relation for the free energy of the two models [24].

The ratio of the susceptibility amplitudes $\mathcal{R}_{\chi}=\Gamma_{4 \mathrm{p}}(+) / \Gamma_{4 \mathrm{p}}(-)=\Gamma_{\mathrm{bw}}(+) / \Gamma_{\mathrm{bw}}(-)$, on the other hand, is not known exactly and different approximations (analytical, series expansions, Monte Carlo data) from different groups are not coherent and differ substantially (for a recent discussion, see the papers [20,26]). It is well known that logarithmic corrections, if they exist, complicate the critical behavior and render the analysis of the critical behavior extremely difficult if possible at all, and the determination of critical exponents from Monte Carlo (MC) and series expansions (SE) became non-trivial. In fact, the determination of critical exponents and corrections to scaling may lead to indecisive conclusions [27].

\footnotetext{
1 We have to note some recent discussion of the weak universality of correlation length amplitudes $[10,11]$ and associated quantities like Binder cumulants $[12,13]$ in the case of anisotropic models.
} 
The purpose of the present paper is to estimate numerically critical amplitudes of the BaxterWu model, which is free of logarithmic corrections, and to compare universal amplitude ratios with the ones available for the 4-state Potts model. It should be emphasized that critical amplitude ratio universality is a non-local property of the renormalization group phase space whereas the critical exponents characterize its behavior only in the vicinity of the corresponding fixed point.

We use the traditional Metropolis MC algorithm to simulate the Baxter-Wu model, and analyze the magnetization and polarization in the ordered phase, and the energy, specific heat and magnetic susceptibility in both phases. We estimate the accuracy of our data by comparing the magnetization, polarization, energy and specific heat to available exact results.

In particular, we use the known results for the energy, specific heat, magnetization and polarization in order to estimate the critical temperature window, i.e., the range of temperatures in which the system on a finite lattice behaves to a very good approximation as on an infinite one. This allows us to estimate the critical ratio of the susceptibility amplitudes in the high- and low-temperature phase with good accuracy, $\mathcal{R}_{\chi}=3.9 \pm 0.1$. The analytical estimate of this ratio, obtained by Delfino and Cardy [28] using some approximation of the exact scattering field theory [29], is only slightly larger, $\mathcal{R}_{\chi}=4.013$. Delfino and Grinza [30] obtained practically the same value, $\mathcal{R}_{\chi}=4.02$, using the same approximation for the Ashkin-Teller model with parameters which correspond to the 4-state Potts model universality class. A recent analysis of the MC and SE data for the 4-state Potts model gives an amplitude ratio in the range of about 6.5(4) $[20,26,31]$. At the same time, the values for the universal combination of amplitudes $R_{C}^{-}=\alpha A_{0} \Gamma_{-} / B_{0}^{2}$ in the low-temperature phase reported in Ref. [28] and Refs. [20,26,31] are 0.00508 and $0.0052(2)$, respectively $\left(A_{0}\right.$ and $B_{0}$ are the specific-heat and magnetization amplitudes). This is in perfect agreement with our estimate $0.00517(7)$ we present here for the Baxter-Wu model.

In the rest of the paper we present the details of our analysis of the amplitude ratios for the Baxter-Wu model. In Section 2 and 3 we first give an overview of known analytical results and previous numerical simulations which support them. We then discuss in Section 4 details of our simulation algorithm realization, including the special choice of the lattice, averaging, etc. Section 5 presents the details of our critical amplitude estimation, and the discussion in Section 6 summarizes our results and touches on some open questions.

\section{Model and exact results}

In this section we summarize those known analytical results for the Baxter-Wu model which will be used in the lattice construction, algorithm realization and data analysis.

\subsection{Model}

The Baxter-Wu model is defined on a triangular lattice, with spins $\sigma_{i}= \pm 1$ located at the vertices. The three spins forming a triangular face are coupled with a strength $J$, and the Hamiltonian reads

$$
\mathcal{H}=-J \sum_{\text {faces }} \sigma_{i} \sigma_{j} \sigma_{k},
$$

where the summation extends over all triangular faces of the lattice, both pointing up and down. 


\subsection{Self-duality and critical temperature}

The model is self-dual as found by Wood and Griffiths [32] and Merlini and Gruber [33], who applied the Kramers-Wannier construction developed for the square-lattice Ising model, and showed that both models, Ising on the square lattice and Baxter-Wu on the triangular lattice, share the same self-dual temperature (see also the book [24]). The partition function reads

$$
Z=\sum_{\sigma} \exp \left[\beta \sum_{\text {faces }} \sigma_{i} \sigma_{j} \sigma_{k}\right],
$$

where $\beta=J / k_{B} T$. The dimensionless free energy per site is

$$
f / k_{B} T \equiv \psi(\beta)=-\lim _{N \rightarrow \infty} N^{-1} \ln Z,
$$

where $N$ is the number of lattice sites. It satisfies the duality relation

$$
\psi(\beta)=\psi\left(\beta^{*}\right)+\ln \left(\sinh 2 \beta^{*}\right),
$$

where

$$
\sinh 2 \beta^{*} \sinh 2 \beta=1 .
$$

This is precisely the duality relation of the square lattice Ising model. The argument of KramersWannier can be applied: if there exists just one critical point, then it must occur when $\beta=$ $\beta_{c}=\beta_{c}^{*}$, where

$$
\sinh 2 \beta_{c}=1, \quad \beta_{c}=\ln (\sqrt{2}+1) / 2 .
$$

\subsection{Ground-state symmetry}

Let $\sigma_{A}, \sigma_{B}, \sigma_{C}$ denote all the spins on the $A, B, C$ sub-lattices, respectively. Any total configuration $\left(\sigma_{A}, \sigma_{B}, \sigma_{C}\right)$ of spins has the same energy as three others. These configurations can be obtained by flipping all spins on two of the sub-lattices. The spin configurations can be grouped in equal-energy sets of four:

$$
\left(\sigma_{A}, \sigma_{B}, \sigma_{C}\right), \quad\left(\sigma_{A},-\sigma_{B},-\sigma_{C}\right), \quad\left(-\sigma_{A}, \sigma_{B},-\sigma_{C}\right), \quad\left(-\sigma_{A},-\sigma_{B}, \sigma_{C}\right) .
$$

The ground state is thus four-fold degenerate: one ferromagnetic state with magnetization (per site) $m=1$ and three ferrimagnetic states with $m=1 / 3$.

\subsection{Exact solution and critical behavior}

Baxter and Wu solved the model at the critical temperature exactly [21-24] and found that the critical value of the energy $e=\langle\mathcal{H}\rangle / N$ is $e_{0}=-\sqrt{2}|J|$, the specific heat $C=d e / d T$ diverges at $T_{c}$ as $[21,22]$

$$
C \propto|t|^{-2 / 3}
$$

and the critical behavior of the magnetization for $\tau \leqslant 0$ is [34]

$$
m \propto|t|^{1 / 12},
$$

where here the reduced temperature is defined as $t=\left(T-T_{c}\right) / T_{c}$. The critical exponents thus take the values $\alpha=2 / 3$ and $\beta=1 / 12$, which is the two-dimensional 4-state Potts model universality class. 


\subsection{Joyce's results for $C$ and $M$}

Joyce established analytic properties of the free energy per spin and found the explicit form [35]

$$
-\frac{f}{k_{B} T}=\frac{2|J|}{k_{B} T}+\ln \Lambda(u),
$$

where

$$
\frac{1}{\Lambda(u)}=\frac{1}{(1+u)^{2}}{ }_{2} F_{1}\left[\frac{1}{2}, \frac{1}{6} ; \frac{4}{3} ; \frac{16 u(1-u)^{2}}{(1+u)^{4}}\right],
$$

with ${ }_{2} F_{1}$ denoting the hypergeometric function and $u=\exp \left(-4|J| / k_{B} T\right)$ such that the critical point value in this variable is $u_{c}=3-2 \sqrt{2}$.

The specific heat critical behavior follows as

$$
\frac{C(t)}{k_{B}}=A_{0}|t|^{-\frac{2}{3}}+A_{1}+A_{2} t|t|^{-\frac{2}{3}}+A_{3}|t|^{\frac{2}{3}}+\mathcal{O}(t),
$$

where

$$
\begin{aligned}
& A_{0}=\frac{2}{9}(\ln (\sqrt{2}+1))^{\frac{4}{3}}=0.187787867 \ldots, \\
& A_{1}=-\frac{1}{2}(\ln (\sqrt{2}+1))^{2}=-0.388409700 \ldots, \\
& A_{2}=\frac{2}{27}(\ln (\sqrt{2}+1))^{\frac{4}{3}}(7 \sqrt{2} \ln (\sqrt{2}+1)-4)=0.295775490 \ldots, \\
& A_{3}=\frac{5}{9}(\ln (\sqrt{2}+1))^{\frac{8}{3}}=0.396723182 \ldots
\end{aligned}
$$

The magnetization reads [36] $(t \leqslant 0)$

$$
m=|t|^{\frac{1}{12}}\left(B_{0}+B_{1}|t|^{\frac{2}{3}}+B_{2}|t|+B_{3}|t|^{\frac{4}{3}}+\mathcal{O}\left(|t|^{\frac{5}{3}}\right)\right),
$$

where

$$
\begin{aligned}
& B_{0}=2^{\frac{3}{8}}(\ln (\sqrt{2}+1))^{\frac{1}{12}}=1.283264709 \ldots, \\
& B_{1}=-\frac{1}{2^{\frac{5}{8}}}(\ln (\sqrt{2}+1))^{\frac{3}{4}}=-0.589829210 \ldots, \\
& B_{2}=\frac{1}{24 \cdot 2^{\frac{5}{8}}}(\ln (\sqrt{2}+1))^{\frac{1}{12}}(4-\sqrt{2} \ln (\sqrt{2}+1))=0.073615269 \ldots, \\
& B_{3}=\frac{1}{2^{\frac{5}{8}}}(\ln (\sqrt{2}+1))^{\frac{17}{12}}=0.5422084696 \ldots
\end{aligned}
$$

Some time later, Baxter [24] found an elegant form for the free energy in terms of an infinite sum of the ratio of some polynomials. The magnetization (and also the polarization defined below in Eqs. (34) and (35)) can be expressed in terms of an infinite product of ratios of polynomials. These expressions may be used to obtain the energy, specific heat, magnetization and polarization with any desired accuracy. The summary of analytical results is presented in this section in a form which is most suitable for the analysis of the data discussed here. 


\section{Previous numerical results}

\subsection{Monte Carlo study of the critical behavior of the Baxter-Wu model}

In course of their simulation study of the influence of quenched impurities on the critical behavior, Novotny and Landau [37] performed for comparison also first Monte Carlo analyses of the pure Baxter-Wu model. They defined the magnetic order parameter $m$ as the root-meansquare (rms) of the magnetization on the three sub-lattices. The polarization order parameter $p$ was defined as the rms average of the two-spin correlation functions for the nearest-neighbor spins between different sub-lattices. They pointed out that the rms rather than the absolute value of the sum of the sub-lattice magnetizations was used because the susceptibilities calculated using the rms definition gave results that were in closer agreement with high- and low-temperature series-expansion results.

Finite-size scaling plots of the order parameters, the magnetization $m L^{\beta_{m} / v}$ and polarization $p L^{\beta_{p} / v}$, as functions of $t L^{1 / v}$ are consistent with $\beta_{m}=\beta_{p}=\beta=1 / 12$ and $v=2 / 3$. In the symmetric phase $\left(T>T_{c}\right)$ those plots show in a log-log representation a decay with slope $-7 / 12$, approaching asymptotic values at very large values of $t L^{1 / v}$. The energy, specific heat, and susceptibility also behave according to the Baxter-Wu predictions.

They simulated lattices with linear size $L$ up to 66 and estimated magnetic susceptibility amplitudes, $\Gamma_{\mathrm{bw}}(+)=0.03(2)$ and $\Gamma_{\mathrm{bw}}(-)=0.010(5)$, and polarization amplitudes, $\Gamma_{\mathrm{bw}}^{p}(+)=$ $0.06(3)$ and $\Gamma_{\mathrm{bw}}^{p}(-)=0.04(2)$. Therefore, the ratio of amplitudes is about 1.5-3 with an uncertainty of about 50 per cent.

\subsection{Monte Carlo renormalization-group study of the Baxter-Wu model}

In their Monte Carlo renormalization-group (MCRG) analysis, Novotny, Landau, and Swendsen [38] did not find any evidence for logarithmic corrections in the Baxter-Wu model on lattices with sizes $21 \times 21,42 \times 42$, and $147 \times 147$. For the RG eigenvalues they estimated $y_{T}=1.48 \pm 0.03, y_{h}=1.875 \pm 0.003$, and results for $y_{3}$ were consistent with the Barber ansatz [39] $y_{3}=7 / 8$, based on the renormalization group analysis. The latter exponent is the correction-to-scaling magnetic exponent $y_{\sigma_{2}}=2-x_{\sigma_{2}}$ (see, Refs. [7,9] and the discussion in Ref. [26]), which produces the correction-to-scaling exponent $2 / 3$ in the specific heat (16) and magnetization (21).

\subsection{Conformal invariance studies of the Baxter-Wu model and a related site-coloring problem}

The operator content of the Baxter-Wu model has been discussed by Alcaraz and Xavier [40, 41], who extended the original Bethe ansatz solution of the site-coloring problem and solved numerically the corresponding equations of the transfer matrix for toroidal boundary conditions. They found that the latter problem has the same operator content as the 4-state Potts model. The correction-to-scaling effects seem, however, to correspond to different perturbations of the fixed point of the renormalization group. The authors of Refs. [40,41] claim that the correction-toscaling terms contain only integer powers, like those in the Ising model. 


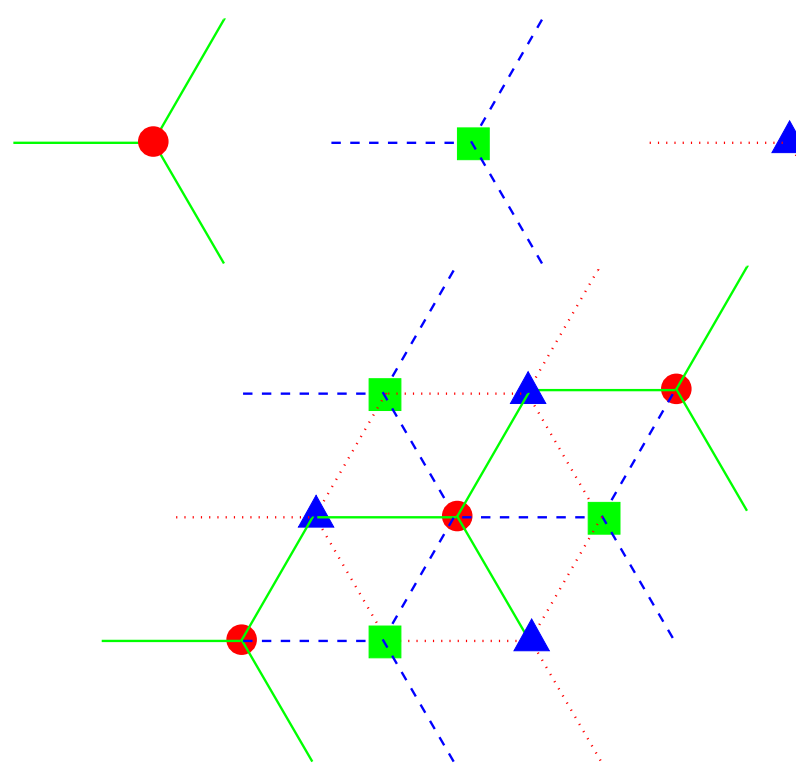

Fig. 1. (Color online.) Top row: Three elements generating the lattice with sites R, G, and B (circle, box, and triangle) and bonds $\mathrm{G}$ (solid line), B (dashed line), and R (dotted line), attached correspondingly. Bottom: The smallest lattice having 3 sites, 3 bonds, and zero sum of site colors and bond colors in any of the three lattice directions under periodic boundary conditions, $L=3$.

\section{Simulations}

\subsection{Lattice choice}

Genuine critical behavior can be observed only in the thermodynamic limit, when the system approaches infinite size. Simulations, on the other hand, are always performed on finite lattices. ${ }^{2}$ Finiteness of the lattice leads to such effects as scaling of thermodynamic quantities with the lattice size at criticality [42], and the shift [43] of the pseudo-critical point. ${ }^{3}$

An additional source for systematic deviations in the simulations is due to the approximation of the aspect ratio which is often overlooked. The central idea is to choose such a form of the finite lattice and boundary conditions for which the number of sites and number of bonds would be the same in either direction, and on both the prime and its dual lattice. ${ }^{4}$ This is the property of the model on the infinite lattice.

We construct the triangular lattice by using the three elements shown in the top row of Fig. 1. We associate one of the three colors with each site and bond, and use the convention that the sum of R (red), G (green), and B (blue) vanishes, or, equivalently, equals to W (white) color.

\footnotetext{
2 At least in the directions of the "space" dimensions, as is the case for the transfer-matrix method where the "time" direction can be taken to infinity.

3 The temperature at which thermodynamic quantities, such as the specific heat and susceptibility, reach a maximum value. The thus defined pseudo-critical temperatures reach $T_{c}$ in the limit of infinite lattice size, and the maximum value of thermodynamic quantities diverges accordingly.

4 It has been demonstrated that such implementation is very helpful in simulations of the bond-percolation problem on the square lattice [44].
} 


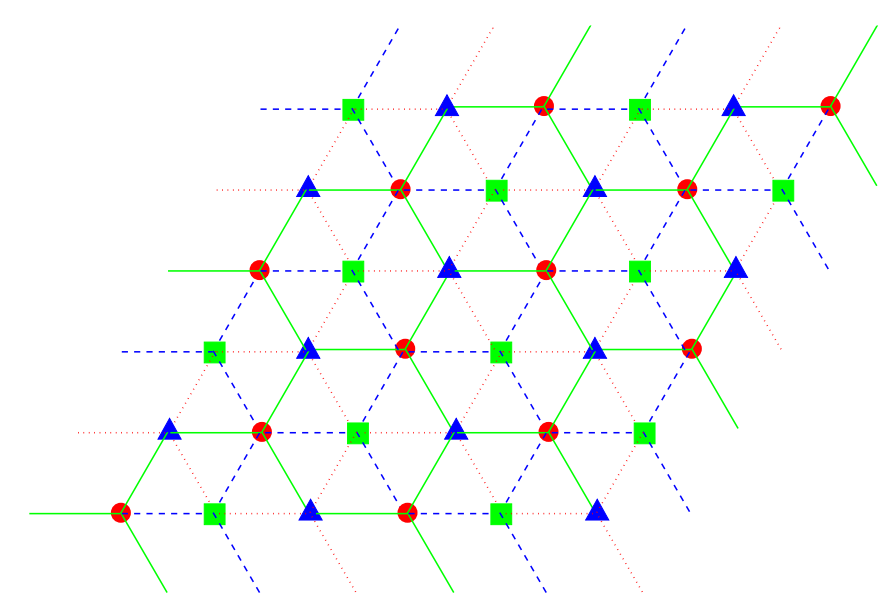

Fig. 2. (Color online.) The next smallest $6 \times 6$ lattice with 6 sites, 6 bonds, and "zero" or "white" colors in any lattice direction under periodic boundary conditions, $L=6$.

The rule of construction is that a site of the same color should never be a neighbor, and the same condition should hold for the bonds. This is the natural partition of the Baxter-Wu lattice on the three sub-lattices. We denote in Fig. 1 an R-site with a circle, a G-site with a box, and a B-site with a triangle. G-bonds are denoted in Fig. 1 with a solid line, B-bonds with a dashed line, and R-bonds with a dotted line.

The minimal lattice size which follows the above mentioned requirements is shown at the bottom of Fig. 1. It contains exactly three sites of different colors and three bonds of different colors in any of the three lattice directions with periodic boundary conditions. The sum of site colors and bond colors is white along any lattice direction (by definition, $R+G+B=W \equiv 0$ ). This $3 \times 3$ lattice can be used as an elementary building block for constructing larger lattices. An example of the next size of the lattice, the $6 \times 6$ lattice, is shown in Fig. 2 .

There are the three sub-lattices for the Baxter-Wu model labeled R, G, and B, therefore the total number of sites (and bonds) should be a multiple of three. The bonds between each two sub-lattices form a hexagonal lattice: for removed sites $\mathrm{R}$ it is composed by the hexagons formed by the red bonds $L_{\mathrm{GB}}$, for removed sites $\mathrm{G}$ by the hexagons formed by the green bonds $L_{\mathrm{RB}}$, and for removed sites B by the hexagons formed by the blue bonds $L_{\mathrm{RG}}$.

Such construction keeps the symmetry of the Baxter-Wu model. In addition, the BaxterWu model is self-dual and our choice of the lattice construction keeps self-duality not only in the thermodynamic limit, but also for any finite size of the lattice. This in turn minimizes the possible influence of the approximation for the aspect ratio [44].

\subsection{Choice of the algorithm}

It is well known that Monte Carlo simulations of the Baxter-Wu model experience strong finite-size effects and an application of the traditional Metropolis algorithm becomes costly due to the critical slowing down. Novotny and Evertz [45] proposed some time ago a cluster algorithm for the Baxter-Wu model. Recently, this algorithm has been extended to the generalized self-dual Baxter-Wu model [46]. The main idea is that the Hamiltonian is invariant under the transformation where all spins on the two sub-lattices are changed. The algorithm fixes the spins 
on one sub-lattice and builds up clusters of correlated spins on the remaining two sub-lattices (see Fig. 2). It is, however, not obvious that such clusters which live on the subspace of possible configurations will percolate at the critical point of the Baxter-Wu model. Indeed, we found that the percolation point of such clusters seems to be shifted a little bit to lower temperatures. As a result this leads to the shift of the curves for some observables. The same effect was found recently for the behavior of Fortuin-Kasteleyn clusters in the $Z_{4}$ spin model [47]. Despite the slowing-down problem, we therefore resorted in our simulations to the traditional Metropolis update algorithm.

\subsection{Metropolis algorithm}

To update the spin configurations with the Metropolis algorithm [48] we calculate the local energy of a spin at position $(j, k)$,

$$
\begin{aligned}
e_{j, k}= & -\sigma_{j, k}\left(\sigma_{j, k-1} \sigma_{j+1, k}+\sigma_{j+1, k} \sigma_{j+1, k+1}+\sigma_{j+1, k+1} \sigma_{j, k+1}+\sigma_{j, k+1} \sigma_{j-1, k}\right. \\
& \left.+\sigma_{j-1, k} \sigma_{j-1, k-1}+\sigma_{j-1, k-1} \sigma_{j, k-1}\right) .
\end{aligned}
$$

If $e_{j, k}>0$, we flip spin $\sigma_{j, k}$. Otherwise we flip the spin only if $\exp \left(2 \beta e_{j, k}\right)$ is not less than a uniformly distributed random number $\in(0,1]$.

\subsection{Averaging over the ensemble}

In simulations, the specific heat can be found from

$$
C=N \frac{1}{k_{B} T^{2}}\left(\left\langle e^{2}\right\rangle-\langle e\rangle^{2}\right)
$$

where the energy per site is calculated as

$$
e=-\frac{1}{N} \sum_{j, k} \sigma_{j, k} \sigma_{j+1, k+1}\left(\sigma_{j+1, k}+\sigma_{j, k+1}\right)
$$

and $N=L^{2}$ denotes the number of sites.

Similarly, the reduced magnetic susceptibility in the low-temperature phase, $\chi_{-}$, can be obtained from

$$
k_{B} T \chi_{-}=N\left(\left\langle m^{2}\right\rangle-\langle m\rangle^{2}\right),
$$

where the magnetization $m$ is computed as the sum of the magnetization over the three sublattices,

$$
m=\left|\sum_{i=1}^{3} m_{i}\right|
$$

with the magnetization per site, $m_{i}$, of the sub-lattice $i$ given by

$$
m_{i}=\frac{1}{N} \sum_{L_{i}} \sigma_{j, k}
$$

In the analysis, we actually calculated the magnetization in an alternative way as [37]

$$
m_{s}=\sqrt{\sum_{i=1}^{3} m_{i}^{2}}
$$


since it leads to more accurate results as was already mentioned in Ref. [37] and will be discussed in more detail below.

The magnetic susceptibility in the high-temperature phase is computed as

$$
k_{B} T \chi_{+}=N\left\langle m^{2}\right\rangle \text {. }
$$

The polarization $p$ per site follows as the sum of the polarizations

$$
p_{i}=\frac{1}{N} \sum_{L_{j k}} \sigma_{j, k}\left(\sigma_{j, k-1}+\sigma_{j+1, k+1}+\sigma_{j-1, k}\right)
$$

between two sub-lattices $j$ and $k$ :

$$
p=\left|\frac{1}{3} \sum_{i=1}^{3} p_{i}\right|
$$

We compute the average of a quantity $A$ (where $A$ is one of $e, e^{2}, e^{4}, m, m^{2}, m^{4}, m_{s}, m_{s}^{2}, m_{s}^{4}$, $\left.p, p^{2}, p^{4}\right)$ as a sum over $N_{\mathrm{av}}$ steps,

$$
\langle A\rangle=\frac{1}{N_{\mathrm{av}}} \sum_{n=1}^{N_{\mathrm{av}}} A_{n} .
$$

Before averaging, we equilibrate the system with $N_{\text {relax }}$ MC steps. Typically, $N_{\text {relax }}=10^{5}-10^{6}$ and $N_{\mathrm{av}}=10^{6}-10^{7}$.

\subsection{Dual reduced temperatures}

We compute thermodynamic quantities at the reduced temperature values $\tau$ and $\tau^{*}$ connected via the duality relation (9) which can be written in the form

$$
\tanh \beta^{*}=e^{-2 \beta} \text {. }
$$

The reduced temperatures $\tau$ and $\tau^{*}$ are defined as

$$
\begin{aligned}
\tau & =\frac{T-T_{c}}{T}=1-T_{c} / T, \quad \tau>0, \\
\tau^{*} & =\frac{T^{*}-T_{c}}{T^{*}}=1-T_{c} / T^{*}, \quad \tau^{*}<0 .
\end{aligned}
$$

Due to (36) the reduced temperatures are related by

$$
\tau=1+\frac{1}{2 \beta_{c}} \ln \left\{\tanh \left[\beta_{c}\left(1-\tau^{*}\right)\right]\right\}, \quad \tau^{*}<0 .
$$

In some formulas we also employ the reduced temperature $t=\frac{T-T_{c}}{T_{c}}$ as in Joyce's papers [35, 36] and in Eqs. (16), (21), which is related to $\tau$ by $t \approx \tau+\tau^{2}+\mathcal{O}\left(\tau^{3}\right)$.

\section{Results}

In this section we employ natural units in which $J=k_{B}=1$ and first define the temperature region window in which we would fit our data. We use the exact knowledge of the specific-heat behavior for that purpose. We demonstrate how reliable the fits to the data are. 


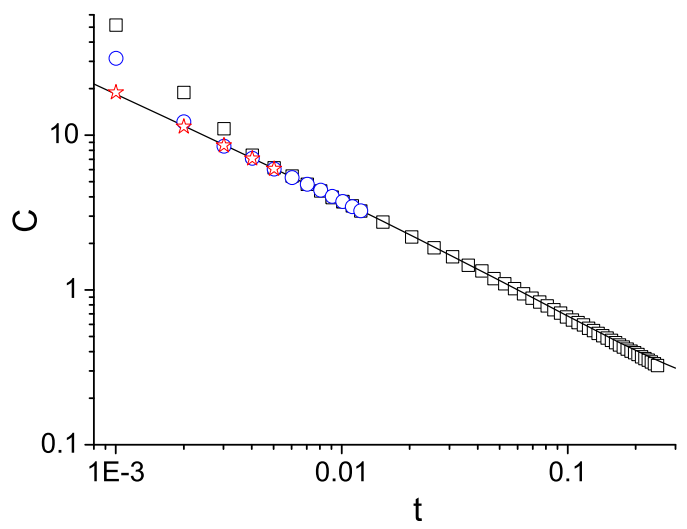

Fig. 3. Specific heat in the high-temperature phase for system sizes $L=162$ (open boxes), $L=243$ (open circles), $L=363$ (stars), and the four-term approximation Eqs. (16)-(20) to the exact solution (solid line). Error bars are of the size of the data symbols.

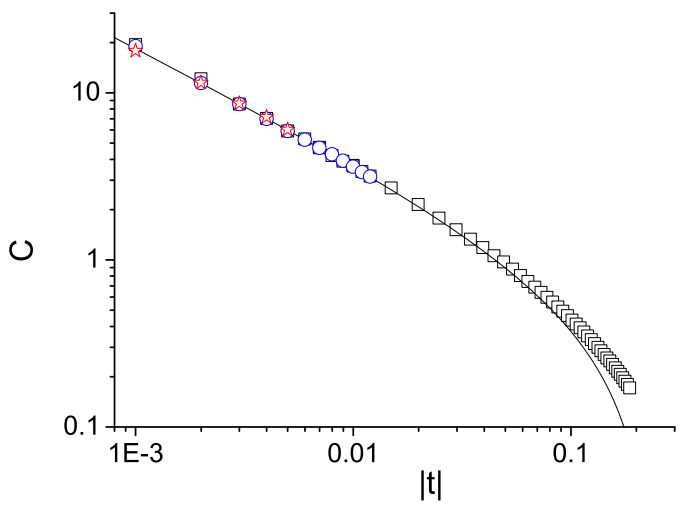

Fig. 4. Specific heat in the low-temperature phase. Symbols and curves are the same as in Fig. 3.

\subsection{Temperature region window}

The specific-heat data in Figs. 3 and 4 exhibit strong finite-size effects close to the critical temperature, that is when the reduced temperature $t$ approaches zero. This is particularly pronounced in the high-temperature phase shown in Fig. 3, where one can see visible deviations of the MC data sets from the exact solution for very small $t$. For temperatures $t>0.003$, however, the shown data sets coincide with each other and with the exact result. For that values of temperature, the correlation length becomes smaller than the system size and the relation $\xi_{0} \propto t^{-v} \ll L$ holds better for larger reduced temperatures.

For large (absolute) values of reduced temperature, the computational data become more and more close to the exact values. At the same time, the solid line, which represents an approximation to the exact solution, starts to diverge from the computational data, because higher-order correction-to-scaling terms, which are not included in the approximation, become more and more important for larger reduced temperatures. So, the critical temperature window is bounded for smaller reduced temperatures $|t|$ by finite-size effects and for larger reduced temperatures $|t|$ by the neglected correction-to-scaling terms in the analysis. 
We have to stress that the left boundary of the temperature window is determined clearly by the nature of the phase transition - it is the temperature at which two length scales coincide: the (randomly) fixed system size and the temperature dependent correlation length. The right boundary of the temperature window is not fixed by any physical reason. It depends on the correction-to-scaling variation with temperature and the number of terms considered in Eq. (16). Here some conventions are necessary. For example, we can define the right boundary as the temperature up to which the first two terms of the full correction set are important. Of course, one can also choose one term or three terms. For some systems or for some particular value of a tuning parameter, it may happen that the first correction-to-scaling term is close to zero, so that in some wide temperature region the system would exhibit the asymptotic scaling behavior, i.e., the correction-to-scaling terms are not important (see the discussion of such an extended scaling in Ref. [49]).

In the case of the Baxter-Wu model, correction-to-scaling terms are not small. The power of correction terms decays slowly with the exponent $1 / 3$ [see Eq. (16)]. The amplitudes of correction terms do not depend on any parameter. So, we have to choose some convention. Using the known powers and amplitudes of correction-to-scaling terms for both the specific heat and magnetization, we can estimate the right edge of the temperature window as that (reduced) temperature for which the relative deviation of the truncated expansion (16), denoted by $C_{N}\left(|t|_{R}\right)$ with $N$ terms, from the exact value of $C\left(|t|_{R}\right)$ is smaller than some $\epsilon,\left|C_{N}\left(|t|_{R}\right) / C\left(|t|_{R}\right)-1\right|<\epsilon$. Fixing some value of $\epsilon$, say $\epsilon \approx 0.001$ (deviation less than one tens of per cent), we can estimate the value of $|t|_{R}$ as a function of the number of correction-to-scaling terms $N$ we want to include in the analysis.

For the data analysis, we will use a combined set of data obtained for the system size $L=363$ when $|t| \leqslant 0.02$ and for the size $L=243$ for larger reduced temperatures $|t|$, if not mentioned otherwise. Both data sets are computed with $N_{\text {relax }}=10^{6} \mathrm{MC}$ steps for relaxation and $N_{\mathrm{av}}=10^{7}$ for averaging.

\subsection{Specific heat}

We define effective amplitudes of the specific heat by

$$
A_{\mathrm{eff}}( \pm)(t)=C(t)|t|^{2 / 3}
$$

where the argument " \pm " refers to the high-temperature (reduced temperature $t$ is positive) and low-temperature phase (reduced temperature $t$ is negative), respectively.

Figs. 5 and 6 show our MC data for the effective specific-heat amplitude in the high- and low-temperature phase, respectively, together with the four-term approximation Eqs. (16)-(20) to the exact solution, which is seen to be in a fairly good agreement with the numerical data.

We fit the following expression for the effective amplitude of the specific heat to the data:

$$
A_{\text {eff }}( \pm)(t)=A_{0}( \pm)+A_{1}( \pm)|t|^{\frac{2}{3}}+A_{2}( \pm) t+A_{3}( \pm)|t|^{\frac{4}{3}}
$$

by varying the temperature region window. Results of the fits are presented in Tables 1 and 2 . The data clearly support in both phases the specific-heat amplitude value $A_{0}=0.1877 \ldots$ rather well. The first correction-to-scaling amplitude $A_{1}=-0.3884$ is estimated less well, but is still precise enough to give support for the theoretically expected value $2 / 3$ of the correction-toscaling exponent. Higher correction-to-scaling terms could not be estimated from the available MC data set. 


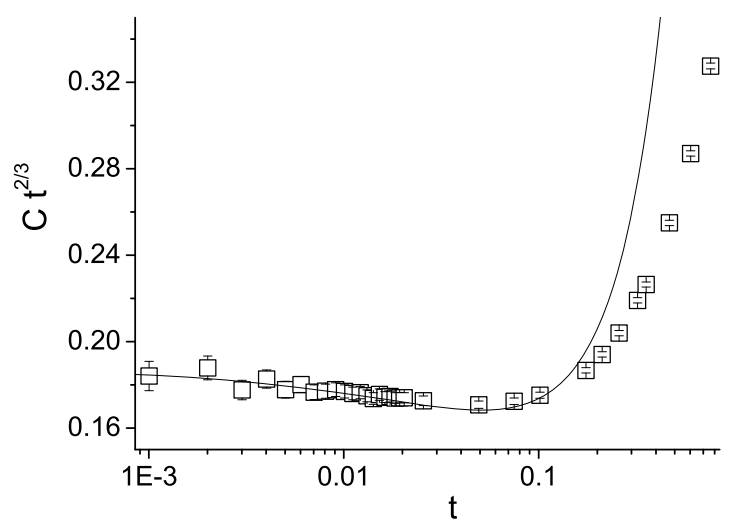

Fig. 5. Effective amplitude of the specific heat in the high-temperature phase. Symbols show combined MC data for $L=243$ (for $t>0.02$ ) and 363 (for $t \leqslant 0.02$ ), and the solid line is the four-term approximation Eqs. (16)-(20) to the exact solution.

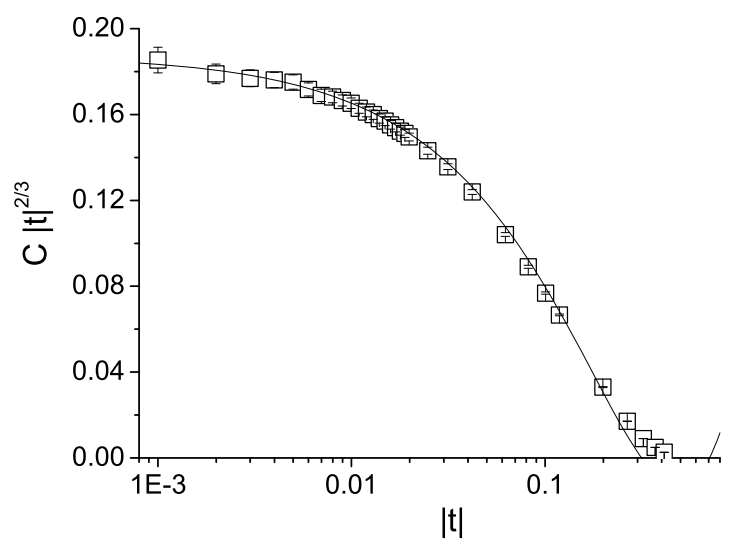

Fig. 6. Effective amplitude of the specific heat in the low-temperature phase. Symbols and curves are the same as in Fig. 5.

\subsection{Energy}

The specific-heat amplitudes are connected with the energy amplitudes. Let us define effective energy amplitudes as

$$
e_{\mathrm{eff}}(+)(t)=\left(e_{+}(t)-e_{0}\right) t^{-\frac{1}{3}}, \quad e_{\mathrm{eff}}(-)(t)=\left(e_{-}(t)-e_{0}\right)|t|^{-\frac{1}{3}}
$$

In the vicinity of the critical point, they can be expanded as [35]

$$
e_{\mathrm{eff}}( \pm)(t)=E_{1}+E_{2}|t|^{\frac{2}{3}}+E_{3} t+E_{4}|t|^{\frac{4}{3}}+\mathcal{O}\left(|t|^{\frac{5}{3}}\right)
$$

with coefficients

$$
\begin{array}{ll}
E_{1}=1.278376401 \ldots, & E_{2}=-0.881371587 \ldots, \\
E_{3}=0.503377046 \ldots, & E_{4}=0.540143046 \ldots
\end{array}
$$


Table 1

Results of fits to the MC data for the effective specific-heat amplitude in the high-temperature phase.

\begin{tabular}{|c|c|c|c|c|}
\hline Fit window & $A_{0}(+)$ & $A_{1}(+)$ & $A_{2}(+)$ & $A_{3}(+)$ \\
\hline exact (Ref. [35]) & $0.1877 \ldots$ & $-0.3884 \ldots$ & $0.2957 \ldots$ & $0.3967 \ldots$ \\
\hline $0.001<t<0.76$ & $0.1878(8)$ & $-0.42(3)$ & $0.91(7)$ & $-0.29(5)$ \\
\hline $0.001<t<0.32$ & $\begin{array}{l}0.1879(12) \\
0.1862(8)\end{array}$ & $\begin{array}{l}-0.43(5) \\
-0.32(2)\end{array}$ & $\begin{array}{l}0.93(17) \\
0.58(3)\end{array}$ & $\begin{array}{l}-0.31(14) \\
0\end{array}$ \\
\hline $0.003<t<0.76$ & $\begin{array}{l}0.1865(8) \\
0.1827(6)\end{array}$ & $\begin{array}{l}-0.38(3) \\
-0.24(1)\end{array}$ & $\begin{array}{l}0.82(6) \\
0.46(1)\end{array}$ & $\begin{array}{l}-0.24(4) \\
0\end{array}$ \\
\hline $0.003<t<0.32$ & $\begin{array}{l}0.1852(12) \\
0.1849(6)\end{array}$ & $\begin{array}{l}-0.31(6) \\
-0.30(1)\end{array}$ & $\begin{array}{l}0.59(18) \\
0.54(2)\end{array}$ & $\begin{array}{l}-0.04(15) \\
0\end{array}$ \\
\hline $0.001<t<0.025$ & $\begin{array}{l}0.1893(22) \\
0.1846(11)\end{array}$ & $\begin{array}{l}-0.52(16) \\
-0.16(2)\end{array}$ & $\begin{array}{l}1.2(5) \\
0\end{array}$ & $\begin{array}{l}0 \\
0\end{array}$ \\
\hline $0.001<t<0.015$ & $0.1860(15)$ & $-0.20(4)$ & 0 & 0 \\
\hline $0.001<t<0.01$ & $0.1870(23)$ & $-0.24(7)$ & 0 & 0 \\
\hline
\end{tabular}

Table 2

Results of fits to the MC data for the effective specific-heat amplitude in the low-temperature phase.

\begin{tabular}{|c|c|c|c|c|}
\hline Fit window & $A_{0}(-)$ & $A_{1}(-)$ & $A_{2}(-)$ & $A_{3}(-)$ \\
\hline exact (Ref. [35]) & $0.1877 \ldots$ & $-0.3884 \ldots$ & $0.2957 \ldots$ & $0.3967 \ldots$ \\
\hline $0.001<|t|<0.4$ & $\begin{array}{l}0.1895(6) \\
0.1972(11)\end{array}$ & $\begin{array}{l}-0.457(28) \\
-0.863(22)\end{array}$ & $\begin{array}{r}0.615(88) \\
-0.679(30)\end{array}$ & $\begin{array}{l}1.05(7) \\
0\end{array}$ \\
\hline $0.003<|t|<0.4$ & $0.1899(7)$ & $-0.45(29)$ & $0.565(88)$ & $1.01(7)$ \\
\hline $0.001<|t|<0.2$ & $\begin{array}{l}0.1879(7) \\
0.1932(2)\end{array}$ & $\begin{array}{l}-0.351(40) \\
-0.718(24)\end{array}$ & $\begin{array}{l}1.03(15) \\
0.411(41)\end{array}$ & $\begin{array}{l}1.47(15) \\
0\end{array}$ \\
\hline $0.003<|t|<0.2$ & $\begin{array}{l}0.1874(7) \\
0.1945(9)\end{array}$ & $\begin{array}{l}-0.321(39) \\
-0.749(24)\end{array}$ & $\begin{array}{c}1.31(15) \\
-0.461(40)\end{array}$ & $\begin{array}{l}1.55(14) \\
0\end{array}$ \\
\hline $0.001<|t|<0.1$ & $\begin{array}{l}0.1903(6) \\
0.1889(3)\end{array}$ & $\begin{array}{l}-0.587(22) \\
-0.529(4)\end{array}$ & $\begin{array}{l}0.122(45) \\
0\end{array}$ & $\begin{array}{l}0 \\
0\end{array}$ \\
\hline $0.003<|t|<0.1$ & $\begin{array}{l}0.1908(7) \\
0.1888(3)\end{array}$ & $\begin{array}{l}-0.605(23) \\
-0.528(4)\end{array}$ & $\begin{array}{l}0.156(46) \\
0\end{array}$ & $\begin{array}{l}0 \\
0\end{array}$ \\
\hline $0.001<|t|<0.025$ & $\begin{array}{l}0.1888(10) \\
0.1895(4)\end{array}$ & $\begin{array}{l}-0.482(72) \\
-0.541(9)\end{array}$ & $\begin{array}{l}0.19(23) \\
0\end{array}$ & $\begin{array}{l}0 \\
0\end{array}$ \\
\hline $0.003<|t|<0.025$ & $\begin{array}{l}0.1875(13) \\
0.1897(4)\end{array}$ & $\begin{array}{l}-0.398(82) \\
-0.544(8)\end{array}$ & $\begin{array}{l}0.44(25) \\
0\end{array}$ & $\begin{array}{l}0 \\
0\end{array}$ \\
\hline $0.001<|t|<0.01$ & $0.1885(11)$ & $-0.511(30)$ & 0 & 0 \\
\hline
\end{tabular}

Fig. 7 shows the effective amplitude of the energy in the low-temperature phase together with the four-term series expansion (43), (44). Fits to the energy amplitudes are shown in Table 3, which clearly support the first two coefficients in (44). Fits to the effective energy amplitude in the high-temperature phase look very similar. 


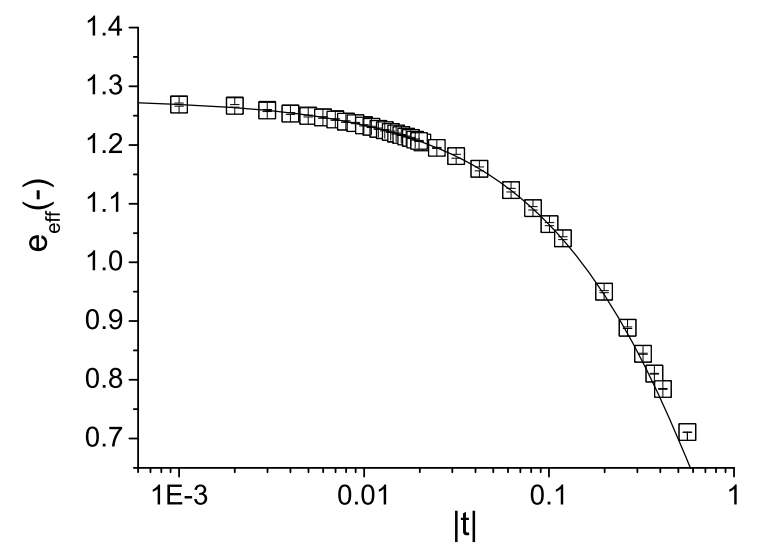

Fig. 7. Effective amplitude of the energy in the low-temperature phase. Symbols are MC data and the solid line is the four-term series expansion (43), (44).

Table 3

Results of fits to the MC data for the effective energy amplitude in the low-temperature phase.

\begin{tabular}{|c|c|c|c|c|}
\hline Fit window & $E_{1}(-)$ & $E_{2}(-)$ & $E_{3}(-)$ & $E_{4}(-)$ \\
\hline exact & $1.2783 \ldots$ & $-0.8813 \ldots$ & $0.5033 \ldots$ & $0.5401 \ldots$ \\
\hline $0.001<|t|<0.56$ & $\begin{array}{l}1.2771(10) \\
1.2946(4)\end{array}$ & $\begin{array}{l}-0.80(4) \\
-1.42(1)\end{array}$ & $\begin{array}{r}0.86(8) \\
-0.68(1)\end{array}$ & $\begin{array}{l}0.99(5) \\
0\end{array}$ \\
\hline $0.001<|t|<0.32$ & $\begin{array}{l}1.2779(14) \\
1.2834(8)\end{array}$ & $\begin{array}{l}-0.84(6) \\
-1.13(2)\end{array}$ & $\begin{array}{r}0.86(8) \\
-0.68(1)\end{array}$ & $\begin{array}{l}0.99(5) \\
0\end{array}$ \\
\hline $0.001<|t|<0.1$ & $\begin{array}{l}1.2795(13) \\
1.2796(6)\end{array}$ & $\begin{array}{l}-0.99(4) \\
-0.99(9)\end{array}$ & $\begin{array}{l}0.01(9) \\
0\end{array}$ & $\begin{array}{l}0 \\
0\end{array}$ \\
\hline $0.001<|t|<0.01$ & $1.2795(17)$ & $-0.99(5)$ & 0 & 0 \\
\hline $0.003<|t|<0.01$ & $1.2779(23)$ & $-0.95(6)$ & 0 & 0 \\
\hline
\end{tabular}

\subsection{Magnetization}

From here on we follow the usual convention in the magnetic sector and use the reduced temperature $\tau$ as independent variable in figures and fits.

The magnetization may be estimated using two methods, defined by Eqs. (30) and (32). Fig. 8 shows the ratio of the magnetization to the exact value, computed using both methods. The relative difference reaches $10^{-5}$ close to the left boundary of the critical region window. We also checked that the low- and high-temperature susceptibilities are not very sensitive to the way the magnetization is calculated and Fig. 8 gives preference for using the definition (32) which we use in this paper for the calculation of the magnetization and the magnetic susceptibility. This was mentioned already in the paper [37].

Fig. 9 shows a comparison of MC data for the magnetization with the expansion (21) of the exact result, where the MC data for several lattice sizes are divided by the exact value. We see that the MC data coincide rather well with the exact result down to the reduced temperature $|\tau| \approx$ 0.003 . This value thus defines the left boundary of the critical region window in our subsequent analysis. 


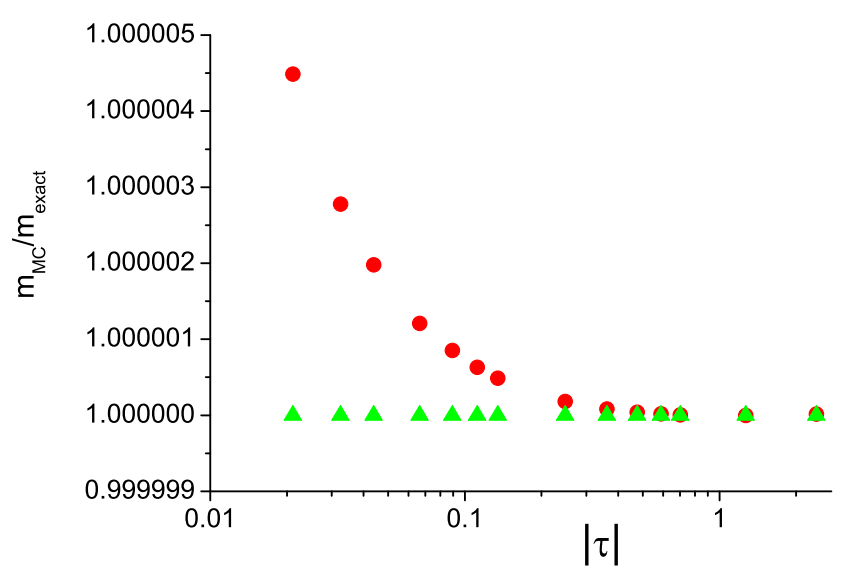

Fig. 8. Ratio of MC data with $L=363$ for the magnetization to the exact result. The magnetization $m_{\mathrm{MC}}$ is computed using Eqs. (30) (circles) and (32) (triangles).

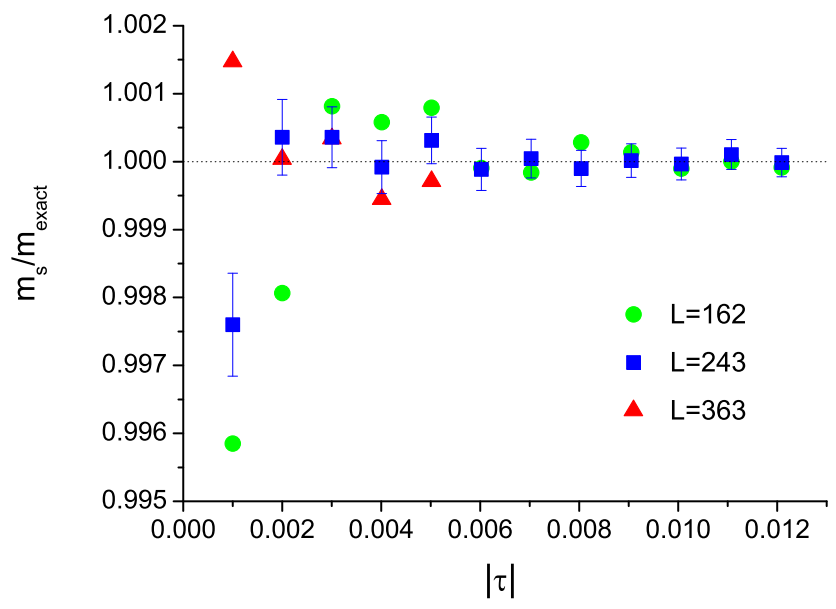

Fig. 9. Ratio of MC data for the magnetization $m_{s}$ to the exact result $m_{\text {exact }}$ for three values of lattice size. Error bars are only shown for the lattice size $L=243$ (open squares).

The data for the effective amplitude $B_{s}=m_{s} /|\tau|^{1 / 12}$ (see Fig. 10) were fit with the expression

$$
B_{s}=B_{0}+B_{1}|\tau|^{2 / 3}+\mathcal{O}(|\tau|),
$$

which, up to this order, agrees with the expansion (21).

For $L=162$, in the temperature window $|\tau| \in[0.003-0.012]$ (compare with Fig. 9), the estimation gives an amplitude of $B_{0}=1.2842$ (4) which is three standard deviations off the exact value $B_{0}=1.28326 \ldots$ For $L=243$, estimated within the appropriate temperature window $|\tau| \in[0.002-0.012]$, the value of the critical amplitude $B_{0}=1.2833(2)$ is in excellent agreement with the exact value. In both cases we found that in the temperature windows it is sufficient to just include the first correction-to-scaling term and we can neglect the second one in the fit. The estimated values of the first correction-to-scaling amplitude are $B_{1}=-0.592(11)$ and $-0.582(6)$ for $L=162$ and $L=243$, respectively, which are in good agreement with the exact 


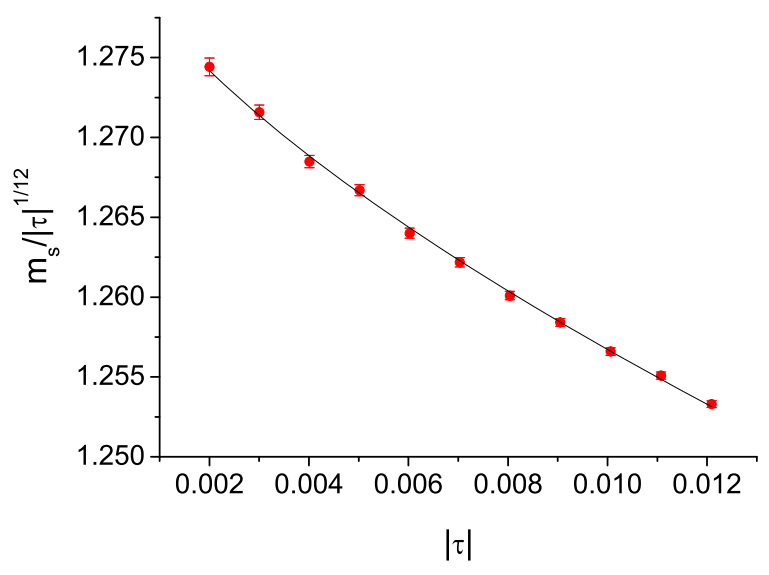

Fig. 10. Effective amplitude of the magnetization: Fit with expression (45) (solid line) to the data for $L=243$ (solid circles).

value $B_{1}=-0.58982 \ldots$ Fig. 10 shows the fit to the effective amplitude of magnetization data for $L=243$.

We also checked the equidistribution of magnetization moments $\left\langle m_{i}^{n}\right\rangle$ and polarization moments $\left\langle p_{i}^{n}\right\rangle$ with $n=1,2,4$ over the three sub-lattices $L_{i}$ and $L_{j k}$ and found it valid within statistical accuracy.

\subsection{Polarization}

The critical amplitude $P_{0}$ of the polarization

$$
P=P_{0}|\tau|^{1 / 12}+\cdots
$$

can be estimated in the same manner as the magnetization critical amplitude $B_{0}$. The final value is $P_{0}=1.2104(3)$, to be compared with the exact value [34]

$$
P_{0}=2^{\frac{15}{8}}(\ln (\sqrt{2}+1))^{\frac{1}{12}} / 3 \approx 1.20987 \ldots
$$

We may also estimate the ratio $B_{0} / P_{0}=1.061$ (1) which agrees perfectly with the exact value $3 \sqrt{2} / 4 \approx 1.06066$.

\subsection{Low-temperature susceptibility}

Let us now come to the main subject of the present paper, the magnetic susceptibility amplitudes which are not known analytically. We can estimate them from our MC data using the same type of analysis we performed for the specific heat and magnetization in the previous subsections. For an additional control of the analysis we compare our MC data with the available series expansions (SE) data. The available SE data [50] are short, however, and could not be used for a reliable estimation of amplitude values.

Fig. 11 shows MC and SE data for the effective susceptibility amplitude $\Gamma_{-}^{\text {eff }}=k_{B} T \chi_{-}|\tau|^{7 / 6}$ in the low-temperature phase as function of $-\tau$. The solid line represents a direct SE data summation, while the dashed line is the Padé approximant of the SE data [51]. MC and SE data 


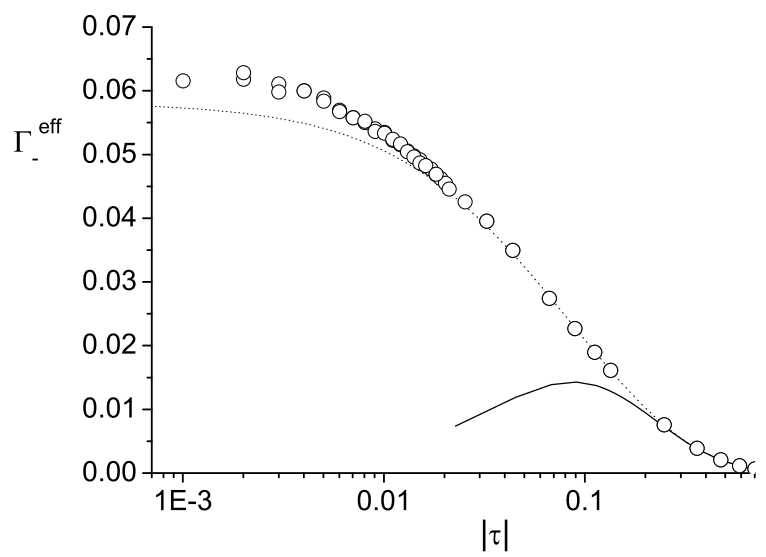

Fig. 11. Effective amplitude $\Gamma_{-}^{\text {eff }}$ of the magnetic susceptibility in the low-temperature phase: MC data (circles), SE data (solid line), Padé approximant to SE data (dashed line).

Table 4

Results of fits to the MC data for the effective low-temperature susceptibility amplitude $\Gamma_{-}^{\text {eff }}$.

\begin{tabular}{|c|c|c|c|c|}
\hline Fit window & $\Gamma_{-}$ & $D_{1}^{-}$ & $D_{2}^{-}$ & $D_{3}^{-}$ \\
\hline $0.002<|\tau|<0.5$ & $0.06814(5)$ & $-0.423(3)$ & $0.491(12)$ & $-0.11(1)$ \\
\hline $0.002<|\tau|<0.25$ & $\begin{array}{l}0.06751(6) \\
0.06800(3)\end{array}$ & $\begin{array}{l}-0.355(6) \\
-0.406(2)\end{array}$ & $\begin{array}{l}0.170(24) \\
0.401(3)\end{array}$ & $\begin{array}{l}0.25(3) \\
0\end{array}$ \\
\hline $0.003<|\tau|<0.25$ & $\begin{array}{l}0.06931(9) \\
0.06893(5)\end{array}$ & $\begin{array}{l}-0.468(7) \\
-0.435(2)\end{array}$ & $\begin{array}{l}0.592(29) \\
0.449(3)\end{array}$ & $\begin{array}{l}-0.15(3) \\
0\end{array}$ \\
\hline $0.002<|\tau|<0.05$ & $\begin{array}{l}0.06804(31) \\
0.06909(10)\end{array}$ & $\begin{array}{l}-0.308(38) \\
-0.444(7)\end{array}$ & $\begin{array}{r}-0.32(22) \\
0.473(20)\end{array}$ & $\begin{array}{l}1.26(34) \\
0\end{array}$ \\
\hline $0.002<|\tau|<0.025$ & $\begin{array}{l}0.06655(8) \\
0.06669(3)\end{array}$ & $\begin{array}{l}-0.273(7) \\
-0.286(1)\end{array}$ & $\begin{array}{l}-0.042(23) \\
0\end{array}$ & $\begin{array}{l}0 \\
0\end{array}$ \\
\hline $0.003<|\tau|<0.025$ & $\begin{array}{l}0.06879(13) \\
0.06715(4)\end{array}$ & $\begin{array}{l}-0.420(9) \\
-0.295(1)\end{array}$ & $\begin{array}{l}0.39(3) \\
0\end{array}$ & $\begin{array}{l}0 \\
0\end{array}$ \\
\hline $0.003<|\tau|<0.011$ & $0.06770(6)$ & $-0.313(2)$ & 0 & 0 \\
\hline $0.004<|\tau|<0.011$ & $0.06801(9)$ & $-0.32(3)$ & 0 & 0 \\
\hline
\end{tabular}

coincide well for $|\tau|>0.01$, and the discrepancy for smaller $|\tau|$ is not surprising due to the short SE data.

The parameters of the fit to the MC data shown in Fig. 11 according to the expression

$$
\Gamma_{-}^{\mathrm{eff}}=\Gamma_{-}+D_{1}^{-}|\tau|^{\frac{2}{3}}+D_{2}^{-}|\tau|+D_{3}^{-}|\tau|^{\frac{4}{3}}
$$

are given in Table 4. Clearly, we can accept as the final and very conservative estimate the value $\Gamma_{-}=0.0681$ (1).

Using the exact values for $A_{0}\left(=A_{0}(-)=A_{0}(+)\right)$ and $B_{0}$, we can estimate from this value the universal ratio

$$
R_{C}^{-}=\alpha A_{0} \Gamma_{-} / B_{0}^{2}=0.00517(7) .
$$




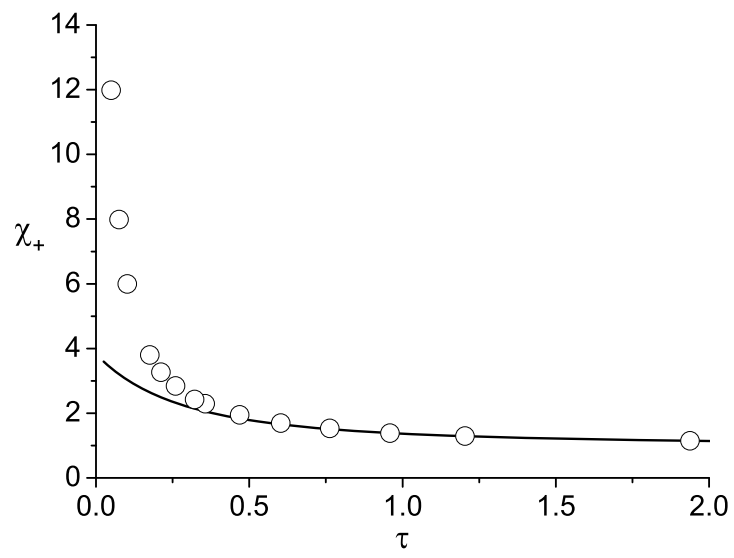

Fig. 12. Magnetic susceptibility $\chi_{+}$in the high-temperature phase: MC data (circles), SE data (line).

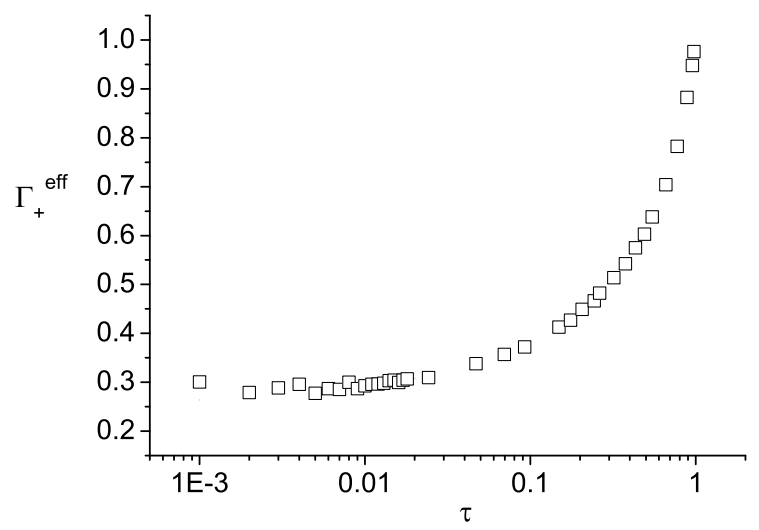

Fig. 13. Effective amplitude of the magnetic susceptibility in the high-temperature phase.

We also estimated the low-temperature critical amplitude of the polarization susceptibility $\Gamma_{-}^{(p)}$ in the same manner as for the magnetic susceptibility. The result is $\Gamma_{-}^{(p)}=0.061(1)$ and $\Gamma_{-} / \Gamma_{-}^{(p)} \approx 1.11$ which appears to be close to the ratio $\left(B_{0} / P_{0}\right)^{2}=1.12499 \ldots$

\subsection{High-temperature susceptibility}

Fig. 12 shows MC and SE [52] data for the susceptibility $\chi_{+}$in the high-temperature phase as function of $\tau$. The data coincide well at large enough reduced temperature $\tau<0.5$ and diverge at small $\tau$ because of the small number of terms in the SE available.

The effective amplitude of the high-temperature susceptibility is shown in Fig. 13. Our results of fits of the effective amplitude data with the expression

$$
\Gamma_{+}^{\mathrm{eff}}=\Gamma_{+}+D_{1}^{+} \tau^{\frac{2}{3}}+D_{2}^{+} \tau+D_{3}^{+} \tau^{\frac{4}{3}}
$$

are presented in Table 5. 
Table 5

Results of fits to the MC data for the effective high-temperature susceptibility amplitude $\Gamma_{+}^{\text {eff }}$.

\begin{tabular}{|c|c|c|c|c|}
\hline Fit window & $\Gamma_{+}$ & $D_{1}^{+}$ & $D_{2}^{+}$ & $D_{3}^{+}$ \\
\hline \multirow[t]{2}{*}{$0.002<|\tau|<0.77$} & $0.265(2)$ & $0.79(7)$ & $-0.93(18)$ & $0.79(12)$ \\
\hline & $0.276(1)$ & $0.34(2)$ & $0.25(2)$ & 0 \\
\hline \multirow[t]{2}{*}{$0.002<|\tau|<0.43$} & $0.267(3)$ & $0.68(12)$ & $-0.59(34)$ & $0.53(27)$ \\
\hline & $0.272(1)$ & $0.45(3)$ & $0.09(4)$ & 0 \\
\hline \multirow[t]{2}{*}{$0.002<|\tau|<0.025$} & $0.266(3)$ & $0.74(48)$ & $-0.7(1.5)$ & 0 \\
\hline & $0.265(1)$ & $0.549(3)$ & 0 & 0 \\
\hline $0.004<|\tau|<0.025$ & $0.270(3)$ & $0.52(6)$ & 0 & 0 \\
\hline $0.002<|\tau|<0.01$ & $0.268(4)$ & $0.57(12)$ & 0 & 0 \\
\hline $0.004<|\tau|<0.01$ & $0.269(7)$ & $0.54(18)$ & 0 & 0 \\
\hline
\end{tabular}

Table 6

Universal combinations of critical amplitudes for the two-dimensional models in the 4-state Potts model universality class.

\begin{tabular}{llllllll}
\hline Model & Approach & $A_{+} / A_{-}$ & $\Gamma_{+} / \Gamma_{-}$ & $\Gamma_{T} / \Gamma_{-}$ & $R_{C}^{-}$ & $R_{C}^{+}$ & Ref. \\
\hline 4-state Potts model & analytical & 1.0 & 4.013 & 0.129 & 0.00508 & 0.0204 & {$[27,28]$} \\
& MC & - & $3.14(70)$ & - & $0.0068(9)$ & $0.021(5)$ & {$[54]$} \\
& SE & - & $3.5(4)$ & - & - & - & {$[55]$} \\
& MC and SE & $1.000(5)$ & $6.49(44)$ & $0.154(12)$ & $0.0052(2)$ & $0.0338(9)$ & {$[20]$} \\
Ashkin-Teller model & analytical & - & 4.02 & 0.129 & - & - & {$[30]$} \\
Baxter-Wu model & MC & $0.995(5)$ & $3.9(1)$ & - & $0.00517(7)$ & $0.0201(5)$ & present \\
\hline
\end{tabular}

The final estimate of the high-temperature susceptibility amplitude is $\Gamma_{+}=0.265(5)$. This implies for the universal susceptibility amplitude ratio the central estimate

$$
\mathcal{R}_{\chi} \equiv \Gamma_{+} / \Gamma_{-}=3.9(1),
$$

in very good agreement with the analytical predictions of Refs. [28] and [30].

\section{Discussion}

It is a widely accepted belief that there are four known models in the 4-state Potts model universality class. Besides the 4-state Potts model itself [16], these are the Baxter-Wu model [21], the Ashkin-Teller model with some particular values of parameters [24], and the DebierreTurban model [53] with some particular value of parameter. Table 6 summarizes the current knowledge of universal amplitude ratios for the first three models, where we also included the universal ratio $R_{C}^{+}=\alpha A_{0}(+) \Gamma_{+} / B_{0}^{2}$. There are no estimations made for the Debierre-Turban model. Clearly, the first two estimates for $\Gamma_{+} / \Gamma_{-}$from Monte Carlo simulations and series expansions are systematically smaller than the analytical predictions, also compatible within error bars, due to the higher uncertainty reported in [54]. There are some visible deviations of the result published in [20]. ${ }^{5}$ The analysis of this quantity presented in Ref. [20] is based on the inclusion

\footnotetext{
5 We mistakenly mentioned in $[20,26]$ a wrong estimation of the value of $\Gamma_{+} / \Gamma_{-}$for the Baxter-Wu model.
} 
of logarithmic corrections to scaling (both multiplicative and additive) in the fit. This procedure is a bit risky although one does not have to do something else. At the same time, it is argued in [20] that the universal combination $R_{C}^{-}$should not contain any logarithmic corrections in the effective estimation through the function $R_{C}(|\tau|)^{-}=\alpha(\alpha-1)\left(e_{-}(|\tau|)-e_{0}\right) \chi_{-} / m^{2}(|\tau|)$ and, indeed, there is a good coincidence of results reported in Refs. [20,28], and in the present paper for $R_{C}^{-}$. By analyzing the data in Table 6 , we may conclude that there are definite overestimations of the critical amplitude $\Gamma_{+}$in Ref. [20]. This is possibly due to the large background terms (nonsingular contribution) in the high-temperature susceptibility. We have to note that there is

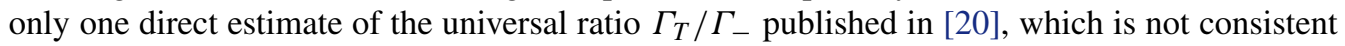
with analytical predictions. More work should be done to clarify this issue.

Finally, we may conclude that our analysis of critical amplitudes of the Baxter-Wu model produces universal amplitude ratios consistent with the analytical results obtained by Cardy and Delfino for the 4-state Potts model [28] and by Delfino and Grinza for the special case of the Ashkin-Teller model [30].

\section{Acknowledgements}

The authors wish to thank Paolo Butera who kindly computed Padé approximants for susceptibility series expansions. We appreciate useful discussions with B. Berche, P. Butera and F. Igloi. This work is supported by the Deutsche Forschungsgemeinschaft (DFG) through Grant No. 436 RUS 17/122/03 and by the Russian Foundation for Basic Research. W.J. gratefully acknowledges support by the Research Academy Leipzig (RAL) and the top-level research area $\mathrm{PbF} 2$ "Mathematical Sciences" of the University of Leipzig.

\section{References}

[1] M.E. Fisher, Phys. Rev. Lett. 16 (1966) 11.

[2] R.B. Griffiths, Phys. Rev. Lett. 24 (1970) 1479.

[3] V. Privman, P.C. Hohenberg, A. Aharony, in: C. Domb, J.L. Lebowitz (Eds.), Phase Transitions and Critical Phenomena, vol. 14, Academic Press, New York, 1991, pp. 1-134, 364-367.

[4] B.M. McCoy, T.T. Wu, The Two-Dimensional Ising Model, Harvard University Press, Cambridge, 1973.

[5] M.P.M. den Nijs, J. Phys. A 12 (1979) 1857; M.P.M. den Nijs, Phys. Rev. B 27 (1983) 1674.

[6] R.B. Pearson, Phys. Rev. B 22 (1980) 2579.

[7] B. Nienhuis, J. Phys. A 15 (1982) 199;

B. Nienhuis, J. Stat. Phys. 34 (1984) 731;

B. Nienhuis, in: C. Domb, J.L. Lebowitz (Eds.), Phase Transitions and Critical Phenomena, vol. 11, Academic Press, London, 1987, p. 1.

[8] V1.S. Dotsenko, Nucl. Phys. B 225 (FS11) (1984) 54.

[9] V1.S. Dotsenko, V.A. Fateev, Nucl. Phys. B 240 (FS12) (1984) 312.

[10] X.S. Chen, V. Dohm, Phys. Rev. E 70 (2004) 056136.

[11] V. Dohm, Phys. Rev. E 77 (2008) 061128.

[12] W. Selke, L.N. Shchur, J. Phys. A 38 (2005) L739;

W. Selke, L.N. Shchur, Phys. Rev. E 80 (2009) 042104.

[13] W. Selke, Eur. Phys. J. B 51 (2006) 223; W. Selke, J. Stat. Mech.: Theor. Exp. (2007) P04008.

[14] G. Delfino, G. Mussardo, JHEP Proceedings of the Workshop on Integrable Theories, Solitons and Duality, IFTUnesp, Sao Paulo, Brazil, PoS unesp2002 (2002) 007, arXiv:hep-th/0302056.

[15] R. Kenna, D.A. Johnston, W. Janke, Phys. Rev. Lett. 96 (2006) 115701;

R. Kenna, D.A. Johnston, W. Janke, Phys. Rev. Lett. 97 (2006) 155702;

R. Kenna, D.A. Johnston, W. Janke, Phys. Rev. Lett. 97 (2006) 169901 (Erratum). 
[16] R.B. Potts, Proc. Cambridge Philos. Soc. 48 (1952) 106.

[17] M. Nauenberg, D.J. Scalapino, Phys. Rev. Lett. 44 (1980) 837.

[18] J.L. Cardy, M. Nauenberg, D.J. Scalapino, Phys. Rev. B 22 (1980) 2560.

[19] J. Salas, A. Sokal, J. Stat. Phys. 88 (1997) 567.

[20] L.N. Shchur, B. Berche, P. Butera, Nucl. Phys. B 811 (2009) 491.

[21] R.J. Baxter, F.Y. Wu, Phys. Rev. Lett. 31 (1973) 1294.

[22] R.J. Baxter, F.Y. Wu, Aust. J. Phys. 27 (1974) 357.

[23] R.J. Baxter, Aust. J. Phys. 27 (1974) 369.

[24] R.J. Baxter, Exactly Solved Models in Statistical Mechanics, Academic Press, New York, 1982.

[25] W. Kinzel, E. Domany, A. Aharony, J. Phys. A 14 (1981) L417.

[26] B. Berche, P. Butera, W. Janke, L.N. Shchur, Comput. Phys. Commun. 180 (2009) 493.

[27] G. Delfino, G.T. Barkema, J.L. Cardy, Nucl. Phys. B 565 (2000) 521.

[28] G. Delfino, J.L. Cardy, Nucl. Phys. B 519 (1998) 551.

[29] L. Chim, A.B. Zamolodchikov, Int. J. Mod. Phys. A 7 (1992) 5317.

[30] G. Delfino, P. Grinza, Nucl. Phys. B 682 (2004) 521.

[31] L.N. Shchur, B. Berche, P. Butera, Europhys. Lett. 81 (2008) 1.

[32] D.W. Wood, H.P. Griffiths, J. Phys. C 5 (1972) L253.

[33] D. Merlini, C. Gruber, J. Math. Phys. 13 (1972) 1814.

[34] R.J. Baxter, M.F. Sykes, M.G. Watts, J. Phys. A 8 (1975) 245.

[35] G.S. Joyce, Proc. R. Soc. London A 343 (1975) 45.

[36] G.S. Joyce, Proc. R. Soc. London A 345 (1975) 277.

[37] M.A. Novotny, D.P. Landau, Phys. Rev. B 24 (1981) 1468.

[38] M.A. Novotny, D.P. Landau, R.H. Swendsen, Phys. Rev. B 26 (1982) 330.

[39] M.N. Barber, J. Phys. A 9 (1976) L171.

[40] F.C. Alcaraz, J.C. Xavier, J. Phys. A 30 (1997) L203.

[41] F.C. Alcaraz, J.C. Xavier, J. Phys. A 32 (1999) 2041.

[42] M.N. Barber, in: C. Domb, J.L. Lebowitz (Eds.), Phase Transitions and Critical Phenomena, vol. 8, Academic Press, London, 1983, pp. 145-266.

[43] D.P. Landau, Phys. Rev. A 13 (1976) 2997.

[44] L.N. Shchur, S.S. Kosyakov, Int. J. Mod. Phys. C 8 (1997) 473.

[45] M.A. Novotny, H.G. Evertz, in: D.P. Landau, K.K. Mon, H.-B. Schüttler (Eds.), Computer Simulations Studies in Condensed-Matter Physics VI, Springer, Berlin, 1993, p. 188.

[46] Y. Deng, W. Guo, J.R. Heringa, H.W.J. Blöte, B. Nienhuis, Nucl. Phys. B 827 (2009) 406.

[47] M. Picco, R. Santachiara, A. Sicilia, J. Stat. Mech.: Theor. Exp. (2009) P04013.

[48] N. Metropolis, A.W. Rosenbluth, M.N. Rosenbluth, A.H. Teller, E. Teller, J. Chem. Phys. 21 (1953) 1087.

[49] P. Butera, M. Pernici, Phys. Rev. B 80 (2009) 054408.

[50] M.G. Watts, J. Phys. A 7 (1974) L85;

M.F. Sykes, M.G. Watts, J. Phys. A 8 (1975) 1469.

[51] Paolo Butera, private communication.

[52] D.W. Wood, H.P. Griffiths, J. Math. Phys. 14 (1973) 1715.

[53] J.-M. Debierre, L. Turban, J. Phys. A 16 (1983) 3571.

[54] M. Caselle, R. Tateo, S. Vinci, Nucl. Phys. B 562 (1999) 549.

[55] I.G. Enting, A.J. Guttmann, Physica A 321 (2003) 90. 